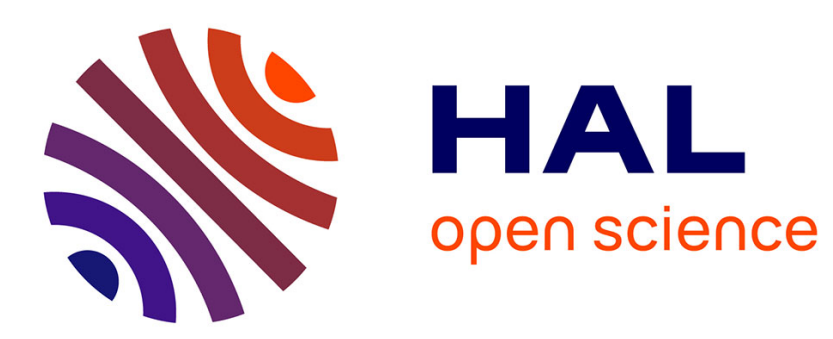

\title{
Statistical correlation between hourly and daily values of solar radiation on horizontal surface at sea level in the Italian climate
}

\author{
U. Amato, B. Bartoli, B. Coluzzi, V. Cuomo, C. Serio, P. Silvestrini
}

\section{- To cite this version:}

U. Amato, B. Bartoli, B. Coluzzi, V. Cuomo, C. Serio, et al.. Statistical correlation between hourly and daily values of solar radiation on horizontal surface at sea level in the Italian climate. Revue de Physique Appliquée, 1986, 21 (3), pp.219-227. 10.1051/rphysap:01986002103021900 . jpa-00245434

HAL Id: jpa-00245434

https://hal.science/jpa-00245434

Submitted on 1 Jan 1986

HAL is a multi-disciplinary open access archive for the deposit and dissemination of scientific research documents, whether they are published or not. The documents may come from teaching and research institutions in France or abroad, or from public or private research centers.
L'archive ouverte pluridisciplinaire HAL, est destinée au dépôt et à la diffusion de documents scientifiques de niveau recherche, publiés ou non, émanant des établissements d'enseignement et de recherche français ou étrangers, des laboratoires publics ou privés. 


\title{
Statistical correlation between hourly and daily values of solar radiation on horizontal surface at sea level in the Italian climate
}

\author{
U. Amato, B. Bartoli, B. Coluzzi, V. Cuomo, C. Serio and P. Silvestrini \\ Dipartimento di Fisica Nucleare, Struttura della Materia e Fisica Applicata, Università di Napoli, \\ 80125 Napoli, Italy
}

(Reçu le 1er août 1984, révisé les 3 mai et 21 novembre 1985, accepté le 21 novembre 1985)

\begin{abstract}
Résumé. - Pour de nombreuses applications pratiques, il est nécessaire de connaître les données horaires du rayonnement solaire. Comme les données horaires ne sont mesurées que dans peu de stations, il faut disposer de méthodes permettant de calculer les valeurs horaires en partant de données journalières. Dans cet article, on analyse les données horaires de stations italiennes et on propose une méthode d'estimation du rayonnement solaire horaire. L'approximation introduite dans l'estimation des valeurs moyennes est comparable à l'erreur statistique. Les courbes obtenues sont indépendantes de la saison et de la station et ne dépendent que du rapport $K$ entre le rayonnement solaire global expérimental et le rayonnement extra-atmosphérique. Elles peuvent être approchées par des paraboles dont les coefficients s'écrivent sous forme d'expressions analytiques simples du rapport $K$. La variance des données expérimentales autour des moyennes a été analysée. La méthode statistique proposée permet de reproduire la distribution de valeurs horaires du rayonnement solaire de façon satisfaisante en partant de valeurs journalières du rayonnement solaire.
\end{abstract}

\begin{abstract}
The knowledge of hourly data of solar radiation is required for many practical applications. Since hourly data are measured only in a few stations, it is quite useful to have methods to calculate hourly values starting from daily data. In this paper we analyse the hourly data measured in Italian stations and propose a method to estimate hourly solar radiation. The approximation introduced by our method in estimating mean values is comparable with its statistical error. The curves we obtain are independent of season and station and depend only on the ratio $K$ of experimental global solar radiation to extraatmospheric radiation. They can be fitted by paraboles whose coefficients can be written as a simple analytical expression of the $K$ ratio. The variance of experimental data around means has been analysed. The statistical method we propose allows to reproduce the distribution of hourly values of solar radiation in a satisfactory way starting from the daily values of solar radiation.
\end{abstract}

\section{Introduction.}

In some cases, the calculation of the performance of solar devices requires the knowledge of solar radiation data on a hourly time basis.

Historical data with this level of detail are seldom available, so that the development of a method to correlate hourly data with daily or monthly averages is of great interest.

Many authors have proposed methods to calculate hourly values of solar irradiation starting from daily data [1-5] and to simulate and forecast solar irradiation on a hourly time basis $[6,7]$. In the first case [1-5] the models give a good estimation of long term means of hourly irradiation as a function of the daily irradiation and of the day length, but do not give any information about the spread of data about the mean. As we shall see, this spread is quite large and, moreover, the time series of hourly values of solar irradiation is not stationary in mean nor in variance [6].

More powerful approaches have been proposed using autocorrelated models of hourly data [6]. In this approach solar irradiance is transformed into a reduced variable which is then modelled as a second order autocorrelated dependent variable. The variable is separated in two kinds of time series according to weather conditions (good or bad) and the autocorrelation coefficients are different for the two cases. This approach yields daily simulations and forecasts one hour ahead but requires the knowledge of the long term average value of hourly solar irradiation for each period of the year $p$ and each hour $h$.

Another model [7] simulates and forecasts the hourly irradiance starting from other variables, as cloud cover, temperature, humidity and atmospheric pressure. 
The knowledge of hourly values is very useful in many practical applications. In some cases the knowledge of averages is sufficient (e.g., for the calculation of long term performances of solar systems [8]), but the knowledge of the actual distribution of hourly values is necessary to describe the behaviour of the system (e.g., overheating effects in solar passive buildings [9]).

Moreover in many stations only daily values are available, so that it is very useful to have methods which allow to calculate average values of hourly solar irradiation and their distribution starting from the values of daily solar irradiance.

In previous papers [10-12] we have shown that the monthly distributions of daily values $K$ (i.e. solar irradiance $G$ normalized to extraatmospheric irradiance $\left.G_{\text {ext }}\right)$ depend only on the monthly average value $\langle K\rangle$ so that the knowledge of $\langle K\rangle$ is sufficient to find the distribution of daily values $K$ and consequently of $G$. Since $\langle K\rangle$ can be estimated starting from the knowledge of the average monthly values of other variables, such as the number of sunshine hours [13,14], this approach allows to build up the distribution of daily values $G$ also in stations where they are not directly measured.

In this paper we propose a similar approach for hourly values of irradiance.

We first estimate average values of hourly irradiances starting from daily values and then we estimate their distribution.

The model is validated for two Italian stations (Genova and Macerata).

\section{Behaviour of the hourly solar irradiation.}

Our analysis has been performed on solar radiation data measured in Genova over a period of nine years (1964-1972) and in Macerata over a period of six years (1964-1969).

In previous papers we have shown that it is convenient to normalize the experimental values of the radiation to extraatmospheric values. We introduce the quantities :

$$
K=\frac{G_{\text {exp }}}{G_{\text {ext }}} \quad X=\frac{X_{\text {exp }}}{X_{\text {ext }}}
$$

where $G_{\exp }$ and $X_{\exp }$ are respectively the daily and hourly experimental values of solar intensity on horizontal surface at sea level, $G_{\text {ext }}$ and $X_{\text {ext }}$ are the corresponding extraatmospheric values. $K$ and $X$ are in fact good candidates as indicators of the physical and meteorological conditions of the atmosphere independently of the locality and season.

In addition, in order to have data independent of the duration of the day (and thus hopefully independent of the season) we have normalized the hour angle ( $\omega=0$ at noon) to the sunrise angle $\omega_{\mathrm{s}}$. Since the behaviour of solar radiation is symmetric around midday, we do not distinguish hours before or after noon, and we assume as time-variable the parameter

$$
H=\left|\frac{\omega}{\omega_{\mathrm{s}}}\right| .
$$

The variables which appear in our analysis are : $K$ : « radiation variable », daily value

$X:$ : radiation variable ", hourly value

$H$ : hour variable

$L$ : locality index

$P$ : period of the year.

The measured values of $X,\left\{X_{j}\right\},(j=1 \ldots N)$ have been grouped as the elements of a 4-index matrix

$$
\begin{gathered}
\left\{X_{i}\right\}_{K h P}^{L} \quad\left(i=1 \ldots M_{K h P}^{L}\right) \\
\sum_{K} \sum_{h} \sum_{L} \sum_{P} M_{K h P}^{L}=N
\end{gathered}
$$

$K=1 \ldots 20$ spans into 20 equal sub-intervals the interval $[0,1]$ of definition of $K$

$h=1 \ldots 16$ spans into 16 equal sub-intervals the interval $[0,1]$ of definition of $H$

$L=\mathrm{G}, \mathrm{M}$ is the index of locality $(\mathrm{G} \equiv$ Genova ; $\mathbf{M} \equiv$ Macerata)

$P=1 \ldots 6$ is an index defining the period of the year, related to solar declination.

Of the 20 sub-intervals of $K$, the first two $(0<K<0.1)$ and the last $5(0.75<K<1)$ are so poorly populated that they have been excluded from our statistical analysis. All the conclusions presented in the following, refer therefore to the 13 sub-intervals for which $0.1<K<0.75$.

First of all, we have computed the average value of the $X_{i}$ belonging to the same matrix element

$$
Y_{K h P}^{L}=\frac{\sum_{i=1}^{M_{K h P}^{L}}\left(X_{i}\right)_{K h P}^{L}}{M_{K h P}^{L}} .
$$

We have then plotted the $Y_{K h P}^{L}$ versus $h$, obtaining a set of $2 \times 20 \times 6$ plots (locality $\times K \times$ period).

Each of them has been best-fitted with a parabolic function using the Least Square Method :

$$
y_{K P}^{L}(H)=a_{K P}^{L}+b_{K P}^{L} H^{2} .
$$

Notice that there was no need of the odd term in these parabolic functions corresponding to the fact that the optical thickness of the atmosphere has its minimum at noon. Some examples are shown in figures 1 and 2.

The distribution of the data points around the curves cannot be attributed only to the statistical errors related to the number of measurements. In addition to instrumental errors, there is a main reason for that : since the time duration of meteorological perturbations is usually much longer than a hour, there is a systematic correlation among the measurements be- 


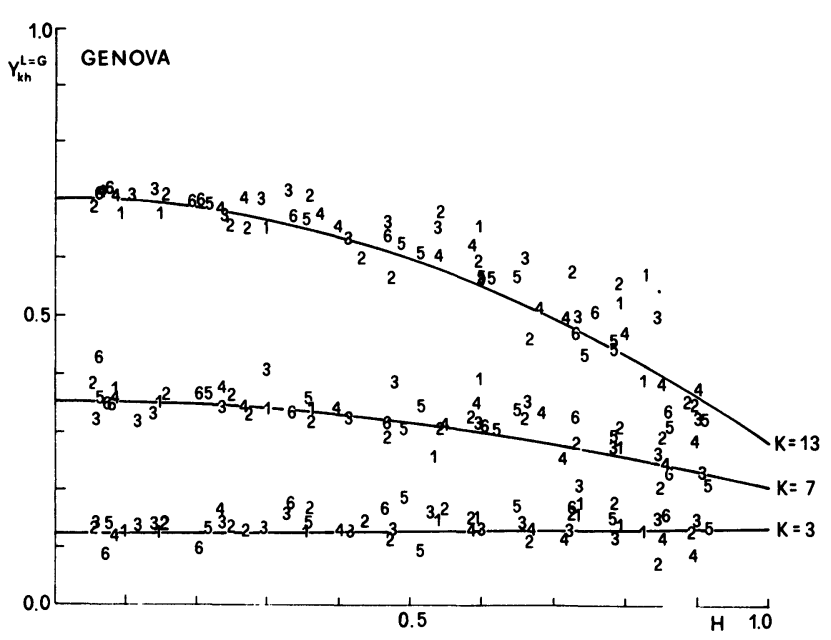

Fig. 1. - Ratio of hourly values of global radiation to extraatmospheric radiation as a function of hour. The data are reported separately for various $K$ values and periods. In the figure we report the data of Genova for $K=0.15$, 0.35 and 0.65 and $P=1,2,3,4,5,6$.

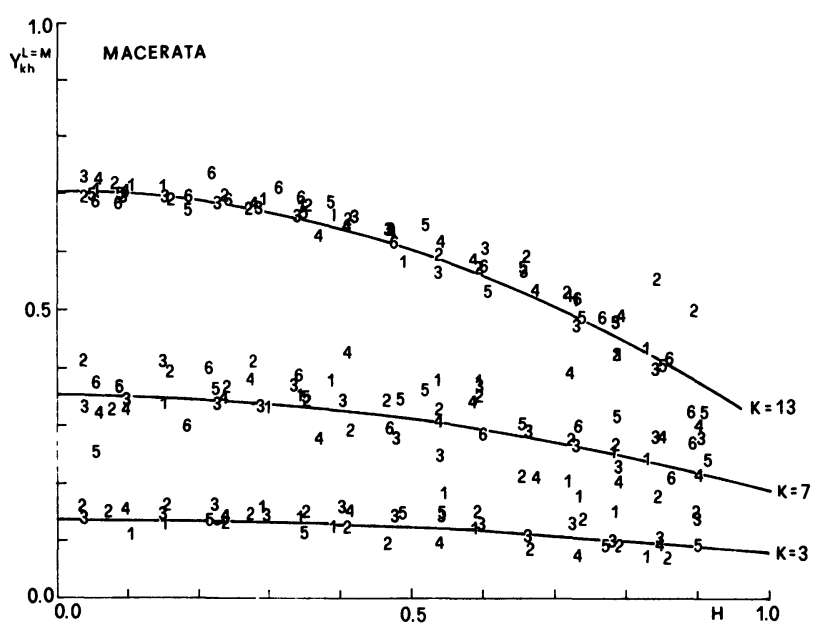

Fig. 2. - As in figure 1 but for Macerata.

longing to the same perturbation. Rather than to the number of measurements, the statistical errors should be thus related to the number of perturbations.

Consequently the error bars $\Delta Y_{K h P}^{L}$ to the data points have been determined as :

where

$$
\Delta y_{K h P}^{L}=c_{K P}^{L} \cdot \sigma_{K h P}^{L}
$$

$$
\begin{gathered}
\sigma_{K h P}^{L}=\left\{\frac{\sum_{i=1}^{M_{K h P}^{L}}\left[\left(X_{i}\right)_{K h P}^{L}-Y_{K h P}^{L}\right]^{2}}{M_{K h P}^{L}}\right\}^{1 / 2} \\
\left(c_{K P}^{L}\right)^{2}=\frac{\sum_{h}\left[y_{K P}^{L}(H)-Y_{K h P}^{L}\right]^{2}}{N_{K P}^{L}}
\end{gathered}
$$

with $N_{K P}^{L}$ being the number of degrees of freedom of the fit $K P L$.

\section{Independence on the period of the year.}

In order to test that the hourly values of solar radiation corresponding to the same value of $K$ and $L$ are independent of the period $P$ of the year, we have used the following procedure :

a) we have computed the quantities

$$
Y_{K h}^{L}=\frac{\sum_{P} \sum_{i=1}^{M_{K h P}^{L}}\left(X_{i}\right)_{K h P}^{L}}{M_{K h}^{L}}, \quad M_{K h}^{L}=\sum_{P} M_{K h P}^{L}
$$

and plotted $Y_{K h}^{L}$ versus $h$, obtaining a set of $2 \times 20$ plots (locality $\times K)$.

The error affecting $Y_{K h}^{L}$ has been computed by propagation of the errors (2.10) affecting $Y_{K h P}^{L}$.

b) We have used the Least Square Method to obtain the best-fit of the $Y_{K h}^{L}$ 's values :

$$
y_{K}^{L}(H)=\alpha_{K}^{L}+\beta_{K}^{L} H^{2} .
$$

Some examples are reported in figures 3 and 4.

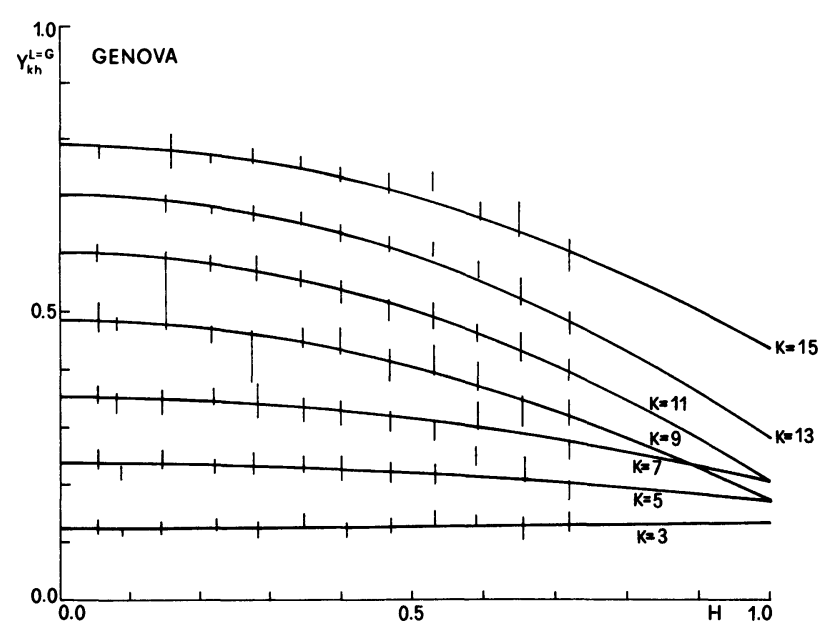

Fig. 3. - Ratio of hourly values of global radiation to extraatmospheric radiation averaged over the period as a function of the hour. In the figure we show the average data of Genova for $K=0.15,0.25,0.35,0.45,0.55,0.65$ and 0.75 together with their best fit.

c) We have submitted to $\chi^{2}$ test the statistical compatibility between each of the $y_{K}^{L}(H)$ and the $Y_{K h P}^{L}(P=1 \ldots 6)$ belonging to the same value of $K$ and $L$.

The results of this analysis (consisting, in total, of $160 \chi^{2}$ tests) are summarized in figure 5 .

As we see, the distribution of the values obtained for the $\chi^{2}$ is in good agreement with the theoretical distribution. We conclude that the behaviour of solar radiation, for a given value of $K$ and $L$, is independent of the period $P$ of the year. 


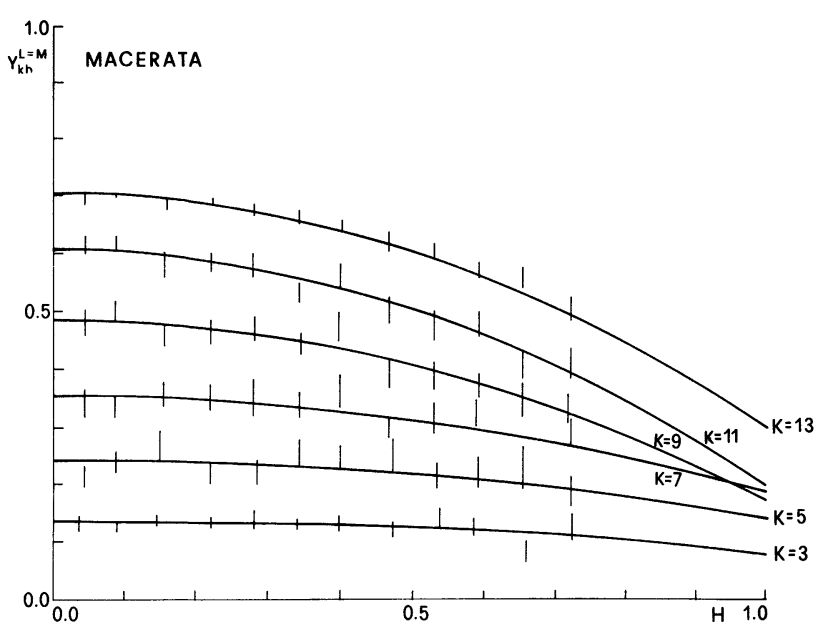

Fig. 4. - As in figure 3 but for Macerata.

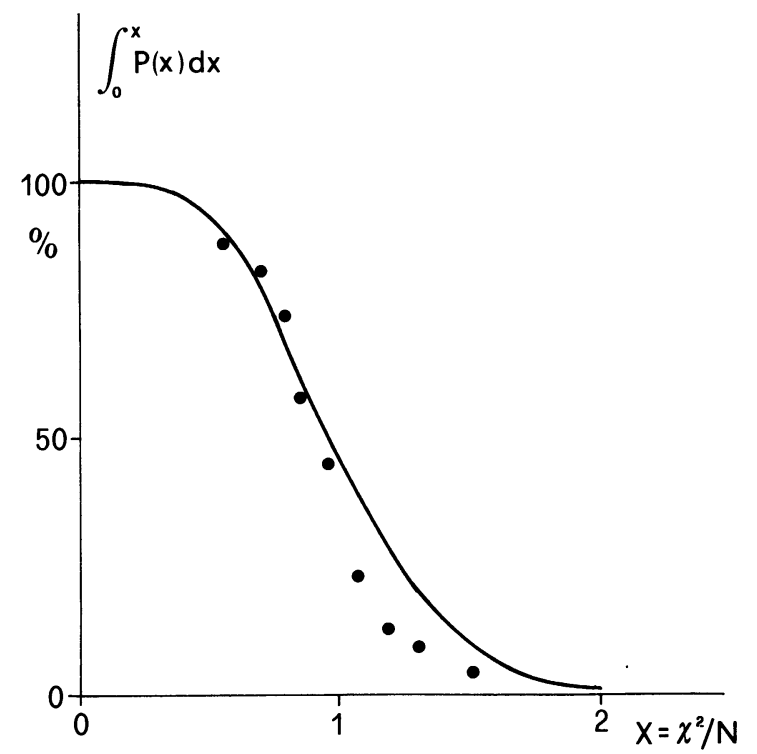

Fig. 5. - For each station we have calculated the best-fit $Y_{K h P}^{L}(H)$ of the mean values of hourly global radiation using the Least Square Method. For each station and each period separately we have submitted to $\chi^{2}$ test the statistical compatibility between every fit $Y_{K}^{L}(H)$ and the experimental data. In this figure we compare the theoretical distribution of $\int_{0}^{X} P(z) \mathrm{d} z\left(z=\chi^{2} / N\right)$ with the actual distribution of $\chi^{2}$ obtained in our case. By this way we have verified that the hourly mean values corresponding to a given locality and a given $K$ are independent of the period.

\section{Independence on the locality.}

In order to check the hypothesis that the availability of hourly solar radiation is essentially independent of the locality, we have used the $\chi^{2}$ test to analyse the agreement between the data of Genova and the curve $3.2 y_{K}^{L}(H)(K=1 \ldots 16)$ obtained starting from the data of Macerata and vice versa. The results of this analysis are summarized in figure 6.
Figure 6a shows the distribution of the values of the $\chi^{2} / N$ 's obtained when the data points $Y_{K h}^{L=G}\left(Y_{K h}^{L=M}\right)$ are compared with $y_{K}^{L=G}(H)\left(y_{K}^{L=M}(H)\right)$ for the same locality. As we see, the agreement is very good, confirming that there is no need of the extra parameter $P$ (see Sect. 3).
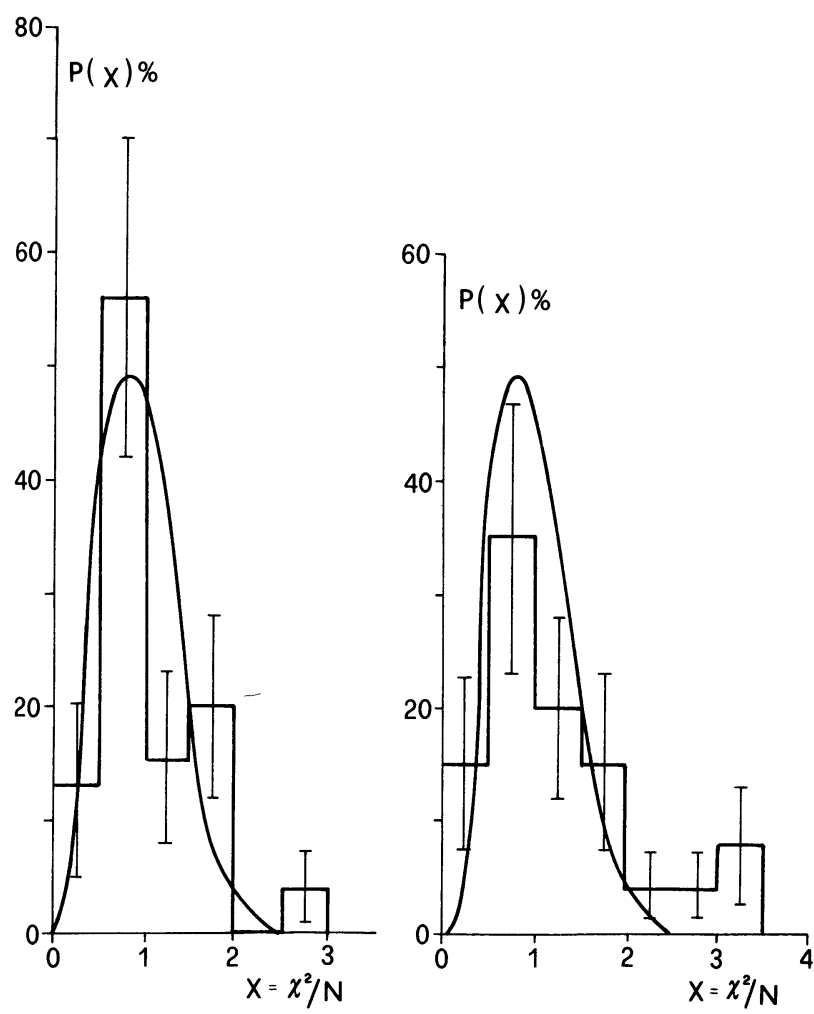

Fig. 6a. - Distribution of the values of the $\chi^{2} / N$ 's obtained when the data points $Y_{K h}^{L=G}\left(Y_{K h}^{L=M}\right)$ are compared with $y_{K}^{L=G}(H)\left(y_{K}^{L=M}(H)\right)$ for the same locality.

Fig. 6b. - Distribution of the values of the $\chi^{2} / N$ 's obtained when the data points of a locality are compared with the curve $y_{K}^{L}(H)$ obtained by LSM fit of the data of the other locality. The analysis assures that the use of the curve of one locality to predict the data points of the other locality does not imply a significant magnification of the purely statistical error.

Figure $6 \mathrm{~b}$ shows the distribution of the values $\chi^{2} / N$ obtained when the data of one locality are compared with the curve $y_{K}^{L}(H)$ obtained starting from the data of the other locality. This distribution is compared with the theoretical one.

We can see that there is a slight amplification of the $\chi^{2} / N$ scale. This fact shows some dependence on the distributions on the locality. This dependence however is so slight that the use of the curve of one locality to predict the data points of the other locality does not imply a significant magnification of the purely statistical errors. 


\section{A compact formula.}

Our analysis has been performed using data from only two stations, so that its generalization requires a check on data from other localities.

On the basis of the data from Genova and Macerata, it results that the mean value of hourly solar radiation intensity normalized to extraatmospheric intensity can be fitted with a parabolic curve :

$$
y_{K}(H)=\alpha_{K}+\beta_{K} H^{2}
$$

where $H$ is the hour angle normalized to the angular length of the solar day (see Eq. (2.2)); $K$ is an index related to daily solar energy normalized to its extraatmospheric value (see Eq. (2.1)).

We have also determined the coefficients $\alpha_{K}$ and $\beta_{K}$ for $K=3 \ldots 15$.

The results obtained are presented in figures 7 and 8. As we see, both $\alpha_{K}$ and $\beta_{K}$ present a rather smooth behaviour as a function of $K$.

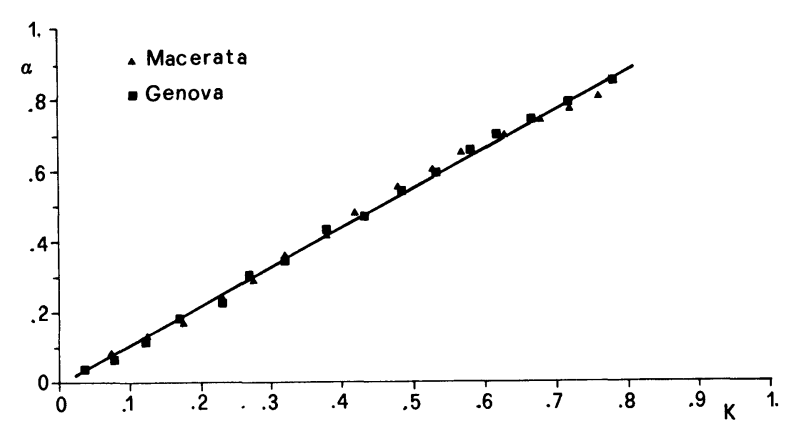

Fig. 7. - The first coefficient of the fit

$$
y_{K}(H)=\alpha_{K}+\beta_{K} H^{2}
$$

as a function of $K$.

In order to put our results in a more convenient form in view of applications, we have determined two curves which represent analytically $\alpha_{K}$ and $\beta_{K}$ respectively.

The result is :

$$
\begin{gathered}
\alpha_{K}=d \cdot K+e \quad d=1.122 \quad e=-0.0093 \\
\beta_{K}=-\exp \left(a+b \cdot K+c \cdot K^{2}\right) \\
a=-5.247 \quad b=15.20 \quad c=-13.24 .
\end{gathered}
$$

The use of the values of $\alpha_{K}$ and $\beta_{K}$ given by equations (5.2) and (5.3) - rather than the numerical values deriving directly from the best-fits to the measured values of $X$ - does not affect drastically the power of equation (5.1) to calculate average value of hourly solar radiation, which actually can be predicted with an error which is at most a factor 1.5 larger than the error affecting the mean value computed on a series of hourly measurements performed in the locality of interest over a period of $6 \div 9$ years.

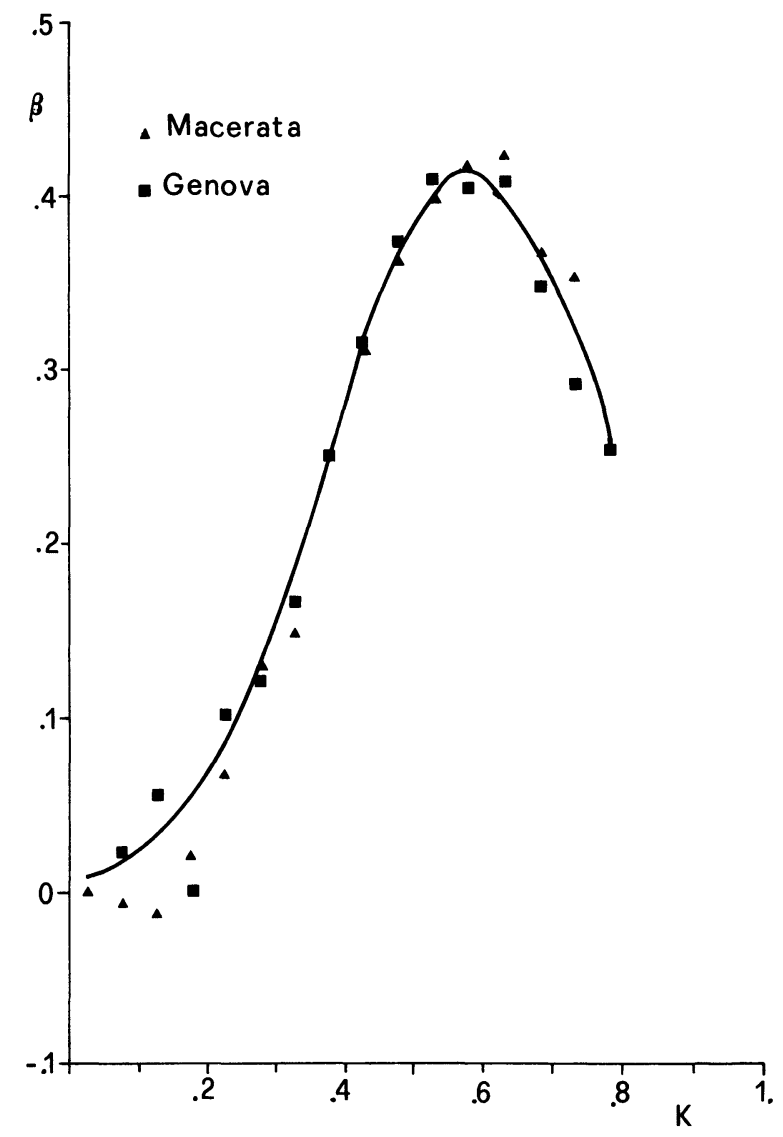

Fig. 8. - The second coefficient of the fit

$$
y_{K}(H)=\alpha_{K}+\beta_{K} H^{2}
$$

as a function of $K$.

\section{Distribution of variances.}

Many solar energy devices also require the knowledge of hourly irradiance distributions around mean values (e.g., threshold systems). In this section we are concerned with the variances of these distributions, since mean and variance are sufficient for performance calculation of most solar systems. The spread of irradiance hourly data about the mean is expected to be larger than that of daily data, since all fluctuations due to microclimatic changes (e.g., atmosphere turbidity, water vapour content, etc.) are more evident on a shorter time basis. Variations could be expected depending on the following three factors :

a) the hour of the day and the period of the year : in fact higher values of irradiance naturally produce larger fluctuations (e.g., for midday-centred hours and summer days);

b) weather conditions : in fact clear days could show small fluctuations due to the absence of clouds. In the same way, completely cloudy days could show small fluctuations, due to the large attenuation of the incoming solar radiation. On the contrary, partly cloudy days are candidate to show the highest varia- 
tions, since different kinds of clouds and different cloudiness conditions during the day are involved.

Analysis has been performed estimating the Root Mean Square deviation $\sigma_{K h}^{L}$, as calculated by equation (2.11), for each locality, $K$ interval, time normalized hour and period of the year.

In the following we divide the full range of the normalized time variable

$$
H^{\prime}=\frac{\omega}{\omega_{\mathrm{s}}} \quad H^{\prime} \in[-1,1]
$$

into 16 equispacied intervals $(h=1 \ldots 16)$.
It results that variances mainly depend on the hour of the day and weather conditions, influence of season being quite smaller. For this reason we have analysed the quantities

$$
\sigma_{K h}^{L}=\frac{\sum_{P} \sum_{i=1}^{M_{K h P}^{L}}\left[\left(X_{i}\right)_{K h P}^{L}-Y_{K h P}^{L}\right]^{2}}{\sum_{P} M_{K h P}^{L}} .
$$

They are shown in table I for Macerata and table II for Genova.

Table I. - Square mean deviation of hourly distributions of irradiance estimated by equation (6.1) for Macerata. $V$ alues are shown for each $K$ interval $(0.1<K<0.80)$ and $H$ interval $(-0.75<H<+0.75$ corresponding to

\begin{tabular}{|c|c|c|c|c|c|c|c|c|c|c|c|c|}
\hline & \multicolumn{12}{|c|}{$\mathrm{H}$} \\
\hline & 3 & 4 & 5 & 6 & 7 & 8 & 9 & 10 & 11 & 12 & 13 & 14 \\
\hline 3 & 0.083 & 0.089 & 0.069 & 0.062 & 0.053 & 0.051 & 0.054 & 0.050 & 0.054 & 0.070 & 0.083 & 0.103 \\
\hline 4 & 0.116 & 0.120 & 0.097 & 0.091 & 0.092 & 0.086 & 0.070 & 0.073 & 0.066 & 0.112 & 0.158 & 0.171 \\
\hline 5 & 0.177 & 0.143 & 0.163 & 0.128 & 0.121 & 0.104 & 0.108 & 0.124 & 0.130 & 0.126 & 0.150 & 0.159 \\
\hline 6 & 0.161 & 0.169 & 0.172 & 0.150 & 0.140 & 0.148 & 0.106 & 0.141 & 0.152 & 0.173 & 0.173 & 0.163 \\
\hline 7 & 0.180 & 0.189 & 0.186 & 0.168 & 0.169 & 0.132 & 0.160 & 0.159 & 0.164 & 0.138 & 0.181 & 0.185 \\
\hline 8 & 0.190 & 0.196 & 0.177 & 0.179 & 0.175 & 0.166 & 0.166 & 0.168 & 0.188 & 0.172 & 0.206 & 0.197 \\
\hline 9 & 0.186 & 0.187 & 0.188 & 0.169 & 0.150 & 0.157 & 0.166 & 0.177 & 0.184 & 0.214 & 0.189 & 0.215 \\
\hline 10 & 0.210 & 0.200 & 0.173 & 0.161 & 0.157 & 0.149 & 0.158 & 0.142 & 0.183 & 0.209 & 0.194 & 0.198 \\
\hline 11 & 0.179 & 0.176 & 0.138 & 0.146 & 0.152 & 0.140 & 0.140 & 0.155 & 0.175 & 0.189 & 0.185 & 0.200 \\
\hline 12 & 0.160 & 0.142 & 0.133 & 0.114 & 0.121 & 0.128 & 0.129 & 0.148 & 0.142 & 0.157 & 0.179 & 0.188 \\
\hline 13 & 0.128 & 0.105 & 0.083 & 0.086 & 0.075 & 0.088 & 0.081 & 0.086 & 0.100 & 0.129 & 0.150 & 0.159 \\
\hline 14 & 0.101 & 0.081 & 0.055 & 0.057 & 0.043 & 0.051 & 0.056 & 0.048 & 0.061 & 0.084 & 0.090 & 0.137 \\
\hline 15 & 0.111 & 0.076 & 0.056 & 0.043 & 0.036 & 0.036 & 0.030 & 0.031 & 0.046 & 0.059 & 0.083 & 0.099 \\
\hline 16 & 0.119 & 0.056 & 0.053 & 0.038 & 0.030 & 0.035 & 0.023 & 0.032 & 0.040 & 0.041 & 0.070 & 0.108 \\
\hline
\end{tabular}
$h=3 \ldots 14)$.

\begin{tabular}{|c|c|c|c|c|c|c|c|c|c|c|c|c|}
\hline & 3 & 4 & 5 & 6 & 7 & 8 & 9 & 10 & 11 & 12 & 13 & 14 \\
\hline 3 & 0.108 & 0.076 & 0.069 & 0.066 & 0.055 & 0.054 & 0.054 & 0.056 & 0.067 & 0.067 & 0.085 & 0.076 \\
\hline 4 & 0.099 & 0.087 & 0.089 & 0.092 & 0.081 & 0.070 & 0.076 & 0.073 & 0.099 & 0.133 & 0.121 & 0.125 \\
\hline 5 & 0.143 & 0.130 & 0.115 & 0.107 & 0.115 & 0.094 & 0.109 & 0.112 & 0.126 & 0.126 & 0.144 & 0.132 \\
\hline 6 & 0.131 & 0.132 & 0.140 & 0.135 & 0.148 & 0.126 & 0.137 & 0.137 & 0.138 & 0.168 & 0.170 & 0.157 \\
\hline 7 & 0.160 & 0.147 & 0.151 & 0.159 & 0.151 & 0.134 & 0.133 & 0.134 & 0.174 & 0.168 & 0.175 & 0.172 \\
\hline 8 & 0.158 & 0.167 & 0.174 & 0.180 & 0.162 & 0.144 & 0.146 & 0.159 & 0.178 & 0.191 & 0.171 & 0.167 \\
\hline 9 & 0.162 & 0.172 & 0.185 & 0.158 & 0.155 & 0.143 & 0.160 & 0.166 & 0.163 & 0.181 & 0.182 & 0.169 \\
\hline 10 & 0.159 & 0.161 & 0.157 & 0.133 & 0.137 & 0.125 & 0.117 & 0.135 & 0.133 & 0.161 & 0.157 & 0.157 \\
\hline 11 & 0.136 & 0.138 & 0.138 & 0.127 & 0.111 & 0.101 & 0.098 & 0.111 & 0.118 & 0.150 & 0.151 & 0.136 \\
\hline 12 & 0.130 & 0.117 & 0.110 & 0.091 & 0.077 & 0.076 & 0.082 & 0.085 & 0.085 & 0.103 & 0.123 & 0.134 \\
\hline 13 & 0.119 & 0.092 & 0.078 & 0.070 & 0.058 & 0.059 & 0.051 & 0.073 & 0.060 & 0.075 & 0.095 & 0.127 \\
\hline 14 & 0.108 & 0.086 & 0.064 & 0.052 & 0.041 & 0.045 & 0.046 & 0.049 & 0.046 & 0.062 & 0.086 & 0.100 \\
\hline 15 & 0.090 & 0.068 & 0.064 & 0.045 & 0.033 & 0.046 & 0.033 & 0.042 & 0.037 & 0.051 & 0.074 & 0.109 \\
\hline 16 & 0.089 & 0.056 & 0.053 & 0.066 & 0.025 & 0.036 & 0.032 & 0.060 & 0.045 & 0.055 & 0.093 & 0.098 \\
\hline
\end{tabular}

Table II. - As table I but for Genova. 
In order to avoid the great quantity of data in tables I and II, for practical uses we have fitted the estimated $\sigma_{K h}^{L}$ values by the following equation, using Least Square Method :

$$
\begin{gathered}
\sigma(K, H)=\sigma_{1}(K) \cdot \sigma_{2}(H) \\
\sigma_{1}(K)=A+B K+C K^{2}+D K^{3} . \\
\sigma_{2}(H)=E+F H+G H^{2}
\end{gathered}
$$

with the following values of the parameters :

$$
\begin{array}{llll}
A=-0.115 & B=1.048 & C=-1.864 & D=0.862 \\
E=0.657 & F=0.084 & G=1.040 .
\end{array}
$$

In figures 9 and 10 we show $\sigma_{1} v$ s. $K$ and $\sigma_{2} v s . H$. Slightly higher values of $\sigma_{1}$ for Macerata are due to a greater instrumentation error in collecting irradiance data.

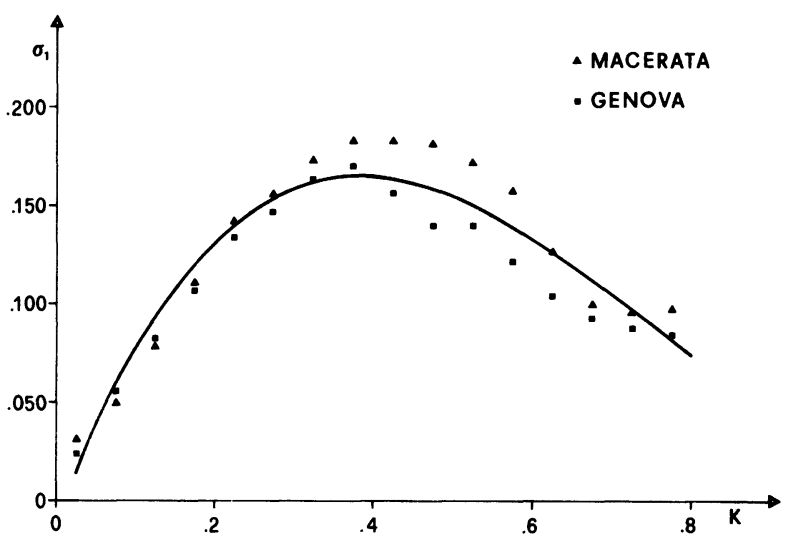

Fig. 9. - Experimental curves $\sigma_{1}$ vs. $K$ for Genova and Macerata. Solid line refers to polynomial fit (Eq. (6.3)).

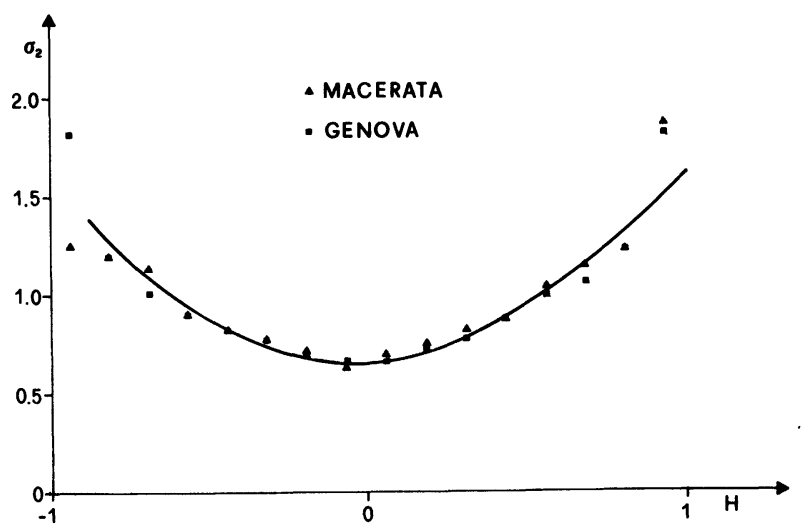

Fig. 10. - Experimental curves $\sigma_{2}$ vs. $K$ for Genova and Macerata. Solid line refers to polynomial fit (Eq. (6.4)).

We want to stress that independence of locality should be verified on data from other localities; however it is desirable from a practical point of view not to take into account typically local environment conditions.

\section{Validation of the analysis.}

In this section we check the goodness of the analysis described till now.

We have analysed experimental distributions of irradiance data for each period of the year $\boldsymbol{P}$ and for each normalized time interval $\boldsymbol{H}$. Correspondingly, we have simulated irradiance data, starting from experimental daily $K$ values.

We assume that normalized hourly irradiance distributions $X$ can be considered as Gaussian with both mean and variance depending on $P$ and $H$. The mean is estimated by parabolic function (Eq. (2.9)) and variance estimate is taken from tables I and II. For each experimental datum $X_{i}$, we have generated the corresponding simulated datum, extracting a random number from a gaussian population, with mean and variance estimated by equation (2.9) and tables I and II. The values so obtained are multiplied by the corresponding $X_{\text {ext }}$ in order to obtain irradiation values $g$. By this way, we obtain 144 experimental and simulated $g$ distributions, corresponding to the six periods in the year, $12 H$ intervals $(|H|<0.75)$ and the two localities.

Then we have compared mean and variance for each couple of distributions, testing if the experimental and the generated ones are statistically compatible.

In figure 11 we show the distribution of the percentage error in estimating mean by our procedure. As we can see, the distribution is centred around 0 , with RMS error being $4.1 \%$, confirming the goodness of the estimate of the mean.

In order to check if variances are statistically equal, we have performed the $F$-test [15] on each couple of

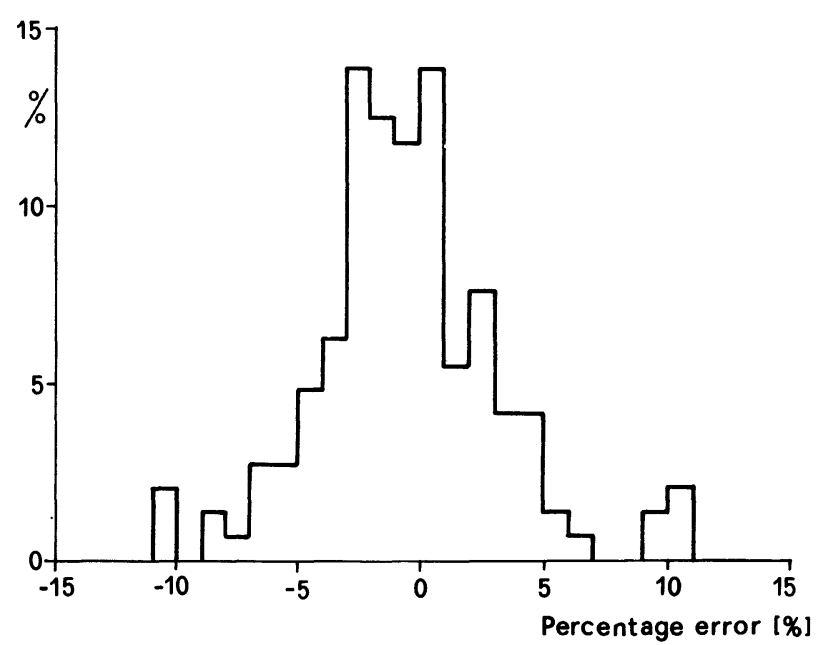

Fig. 11. - Probability density of the percentage error in estimating actual average value of hourly irradiance distributions by equation (2.9). The curve shown refers to 144 samples ( 2 localities $\times 6$ periods $\times 12 H$ intervals). 


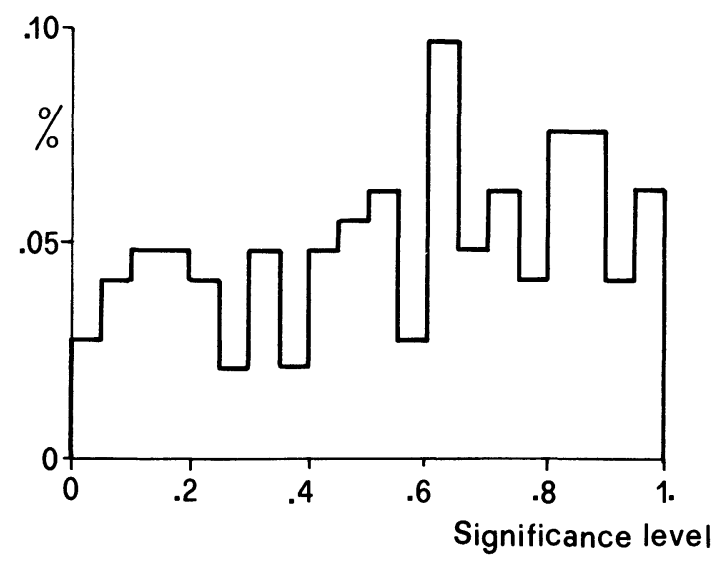

Fig. 12. - Probability density of $F$-test significance level to compare actual variance of hourly irradiance distributions with variance estimated by tables I and II. The curve shown refers to 144 samples ( 2 localities $\times 6$ periods $\times 12 H$ intervals.

distributions. In figure 12 we show the distribution of significance level of $F$-test for all 144 samples; it is quite uniform, confirming that experimental and simulated variances are statistically equal.

In order to check how much of experimental hourly values are reproduced by our method starting from daily data, we have generated for each period the distribution of hourly values of solar global irradiance and we have compared it with the distribution of experimental data $X_{\text {exp }}$.

In figures $13 \mathrm{a}, 13 \mathrm{~b}$ we show experimental and simulated distributions of hourly irradiance (respectively continuous and dashed line) for Macerata and Genova and for sample period 1, independently of $H$. It is evident that the hypothesis of assuming normalized irradiance distributions as normal for each $K$ and $H$ value is correct and does not affect the shape of the distribution of actual irradiance data.

In the same figures, we also show (pointed line) simulated distributions obtained estimating variances by equations (6.2), (6.3) and (6.4). It is evident that the error introduced by LSM Fit of variances is quite negligible. The comparison between experimental distributions and those generated using equation (6.2) shows that also this approach, which is much more convenient than tables I and II, is completely suitable for all practical applications.

\section{Conclusions.}

We have analysed in this paper the distribution of hourly solar radiation on horizontal surface at sea level. We have shown that the mean of the hourly value $X_{\text {exp }}$ of solar radiation - normalized to extraatmospheric radiation $X_{\text {ext }}$

$$
\bar{X}(H)=\frac{X_{\text {exp }}}{X_{\text {ext }}}
$$

depends essentially only on the parameter $K=G_{\text {exp }} /$ $G_{\text {ext }}$ (daily value of solar radiation at ground nor-

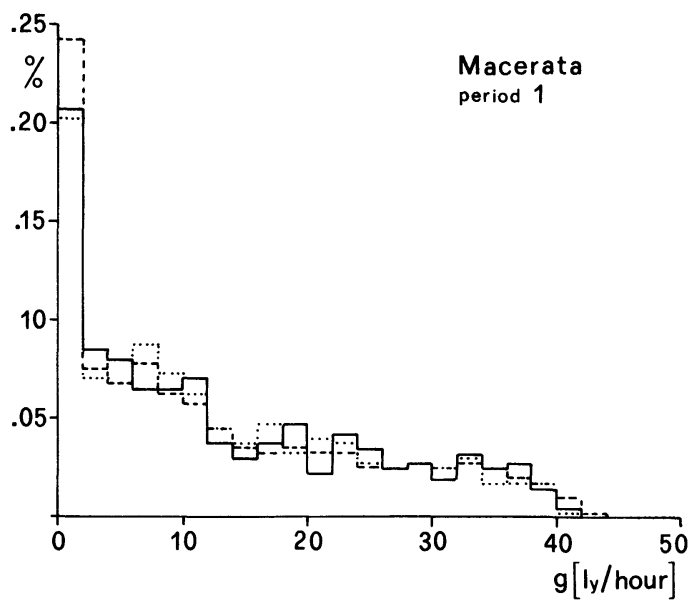

Fig. 13a. - Distribution of hourly irradiance $g$ (units ly/hour ; $1 \mathrm{ly} /$ hour $=11.6 \mathrm{~W} / \mathrm{m}^{2}$ ) for Macerata (period 1 of the year). Solid line refers to experimental data; dashed and pointed lines refer to simulated data, with mean estimated by equation (2.9) and variance estimated respectively by tables I and II and equation (6.2).

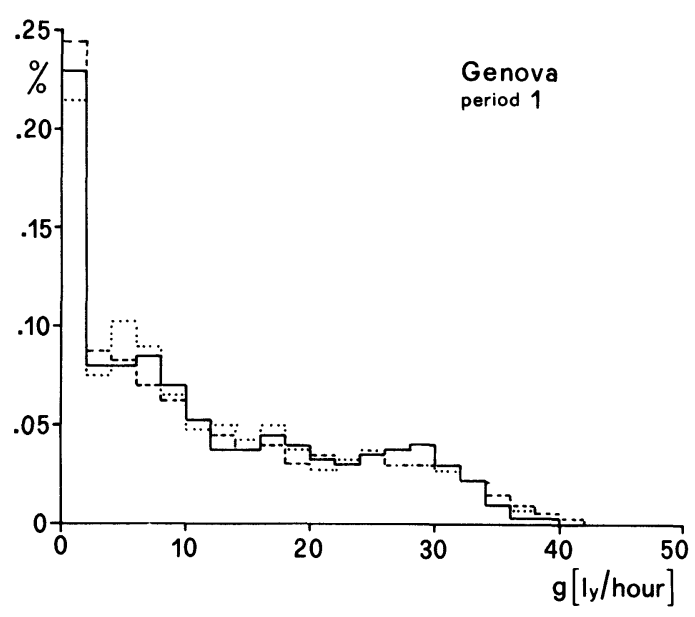

Fig. 13b. - As in figure 13a but for Genova.

malized to extraatmospheric daily radiation) which characterizes the average status of the atmosphere in each day.

In particular, there is no statistically significant dependence on the period of the year, if the hour of the day is normalized to the day length. The dependence on the locality, although statistically detectable on a long period $(\simeq 10$ years) series of hourly data, does not affect appreciably the predictive power of an unique curve independent of locality. In order to put our results in a form which is more confortable from the point of view of applications, we have also determined an analytic expression for $\bar{X}$ :

$$
\begin{aligned}
& \bar{X}(H)=X_{K}(H)=d \cdot K+e-\exp \left(a+b K+c K^{2}\right) H^{2} \\
& a=-5.247 \quad b=15.20 \quad c=-13.24 \\
& d=1.122 \quad e=-0.0093 \text {. }
\end{aligned}
$$


We have shown that also the variance $\sigma^{2}$ of $X_{\text {exp }} /$ $X_{\text {ext }}$ depends only on the parameter $K$ and on the hour of the day normalized to the day length ; in particular it can be considered independent both of locality or period of the year.

An analytic expression for $\sigma^{2}$, useful for all applications, is given by the following :

$$
\begin{aligned}
& \sigma=\left(A+B K+C K^{2}+D K^{3}\right) \cdot\left(E+F H+G H^{2}\right) \\
& A=-0.115 \quad B=1.048 \quad C=-1.864 \quad D=0.862 \\
& E=0.657 \quad F=0.084 \quad G=1.040 \text {. }
\end{aligned}
$$

Finally we have shown that mean and variance are sufficient to reproduce with good accuracy distributions of actual data of hourly irradiance.

\section{References}

[1] Whillier, A., Arch. Meteorol. Geophys. Bioklimatol. Ser. B 7 (2) (1956) 197.

[2] LIU, Y. H. and JORDAN, R. C., Solar Energy 4 (1) (1960) 1.

[3] IQBAL, M., Solar Energy 22 (1) (1979) 87.

[4] Collares-Pereira, M. and Rabl, A., Solar Energy 22 (2) (1979) 87.

[5] WhILlieR, A., Solar Energy 9 (3) (1965) 164.

[6] Boch, G., Bolleau, E. and BéNARd, C., Revue Phys. Appl. 16 (1981) 237.

[7] AstieR, R., DUhamel, Ch. and BÉNARD, C., Revue Phys. Appl. 18 (1983) 29.

[8] Bartoli, B., Coscia, U., Cuomo, V., Fontana, F. and Silvestrini, V., Revue Phys. Appl. 18 (1983) 281.

[9] Amato, U., Coluzzi, B., Cuomo, V., Serio, C. and Troise, G., Appl. Energy 17 (1984) 263.
[10] Bartoli, B., Catalanotti, S., Cuomo, V., Francesca, M., Serio, C., Silvestrini, V. and Troise, G., Nuovo Cimento 2C (1979) 222.

[11] Andrettá, A., Bartoli, B., Coluzzi, B., Cuomo, V., FranCESCA, M. and Serio, C., Nuovo Cimento 4C (1981) 113.

[12] Amato, U., Andretta, A., Bartoli, B., Coluzzi, B., CuOMo, V. and Serio, C., Solar World Congress (Pergamon Press) 1983, p. 2214.

[13] Page, J. K., Proceedings of the UN Conference on New Sources of Energy 4 (1964) 378.

[14] Andretta, A., Bartoli, B., Coluzzi, B., Cuomo, V., Francesca, M. and Serio, C., J. Appl. Met. 21 (10) (1982) 1377.

[15] SchaEfFE, H., The Analysis of Variance (New York) (1959). 